\title{
Application of Genetic Algorithm \& Morphological Operations for Image Segmentation
}

\author{
Mansoor Farooq \\ School of Science \& Technology/Department of Computer Science, Shri Venkatashwara University, U.P, India
}

\begin{abstract}
Image segmentation is an important part of the digital image processing. It is a low level, but difficult task that segments the image into regions of similar attributes. Segmentation simply changes the illustration of the image by dividing the image into similar components something that is more significant and easier to study. Till now there is no fundamental theory or algorithm on the subject of image segmentation. Although, in this paper genetic algorithm and mathematical morphological operations are used for optimizing or detecting homogenous regions of an image. The operation is performed on $16 \times 16$ subimages and the resultant subimages are then combined and the segmented images is produced.
\end{abstract}

Keywords: Genetic Algorithm, Image Segmentation, Mathematical Morphology and Optimization

\section{INTRODUCTION}

Genetic Algorithms GAs are robust methods which can be used to resolve search and optimization problems. Genetic Algorithms (GAs) are basically the natural selection process invented by Charles Darwin where it takes input and computes an output where multiple solutions might be taken. The GAs are designed to simulate processes in natural system necessary for evolution. GA performs efficient search in global spaces to get an optimal solution. A genetic algorithm provides the systematic random search. They provide a simple and almost generic method to solve complex optimization problems. A genetic algorithm is a derivative-free and stochastic optimization method. It needs less prior information about the problems to be solved than the conventional optimization schemes such as the steepest descent method, which often require the derivative of the objective functions. Based on individual fitness value, genetic algorithm uses the operators such as reproduction, crossover and mutation to get the next generation that may contain chromosomes providing better fitness [6]. In GA, the new child or chromosome is obtained from the collective features of their parents, same way a new improved image is obtained that has the same features as of their parent image. [7] GA has the ability to determine optimal number of regions of a segmentation result or to choose some features such as the size of the analysis window or some heuristic thresholds.

The two aspects of genetic algorithm are:

\section{Crossover}

The crossover is genetic process which will combine two chromosomes to produce a new chromosome (offspring). The resultant chromosome may have better characteristics than parents and also new offspring properties come from one parent and some from another.

Example, parent 1 is 00100110110 and parent2 is 11000011110 and after the crossover we will get the output, which contains some part of parent 1 and another from parent 2 i.e. $\mathbf{1 1 0 0 0 0 1 1 1 1 0}$

$00100110110+11000011110=\mathbf{1 1 0 0 0 0 1 1 1 1 0}$

\section{Mutation}

Mutation can be takes place after the crossover get performed. This is to prevent falling all solutions in population into a local optimum of solved problem. The mutation depends on the encoding as well as the crossover. For example when we are encoding permutations, mutation could be exchanging two genes. Mutation changes the new offspring randomly. For binary encoding we can switch a few randomly chosen bits from 1 to 0 or from 0 to 1 . Mutation can then be following [6]:

Original offspring 1- 1101111010011110

Mutated offspring 1- 1100111010011110

Original offspring 2- 1101100100110110

Mutated offspring 2- 1101101100110110

A Simple Genetic Algorithm which includes GA operators.[8]

function GeneticAlgo()

Initialize population;

Calculate fitness function;

While(fitness value != termination criteria)

Selection; Crossover;

Mutation;

Calculate fitness function;

\}

\section{\}}

\section{Mathematical Morphology}

Mathematical morphology is a branch of nonlinear transformation and filtering methodology, in context of mathematics and signal processing. The principle behind the mathematical morphology is that it is based on a formulation of set theory developed by J.Serra and G. Matheron. It is an approach to process digital image based on the geometrical shape. Mathematical morphology can easily be applied on binary images, because it naturally 
forms two set of pixels the object and the background. It The image segmentation algorithms are divided into three has been used for extracting edges and detecting major categories[11][12]

characteristic objects in mobile photogrammetry systems 1. Edge Based

to making maps from images taken from a car, called 2. Region Based

mobile mapping systems [9].

The components of mathematical morphology:

\section{Dilation}

In dilation process, dilation operator called as a structural element is compared with the object to be transformed. The result of transformation appears only if the structural element positioned at a given point touche the object, otherwise it will not. Mathematically, dilation combines two sets using vector addition of set element [10]. Dilation is useful for filling holes in segmented objects.

\section{Erosion}

In erosion process, erosion operator called as a structural element is compared with the object to be transformed. The result of transformation appears only if the structural element is included in the object, otherwise it will not. Mathematically, erosion combines two sets using the vector subtraction of set element [10]. Erosion is useful for removing segmented image objects that are too small to be of interest.

\section{Opening}

In opening process, opening operator called as a structural element is compared with the object to be transformed. The result of the transformation appears only if the structural element is included in the image, then the whole structural element otherwise none of its point will appear. The process of erosion followed by dilation is called opening.

\section{Closing}

In opening process, opening operator called as a structural element is compared with the object to be transformed. The result of the transformation appears only if the structural element is included in the complement of the image, then the whole structural element otherwise not. The process of dilation followed by erosion is called closing.

\section{IMAGE SEGMENTATION}

Image recognition is a process in which digital image is divided into different multiple segments. Image segmentation simply changes the representation of the image into something that is more meaningful and easier to analyze. Pixel clustering and parameter selection are two difficult and important goals of the image segmentation.

Image segmentation has a vast application, including

Face recognition

Finger printer recognition

Iris recognition

Video surveillances

Video compression object based

Medical imaging for detection of tumors etc.

\section{Clustering Based}

\section{Edge Based Segmentation}

Edges carry valuable information which is useful for object classification. Edge-based segmentation is primarily used to look for image discontinuities. The technique is generally applied where changes of gray-level intensity occur in the image. The assumption is that changes occur in the data at the boundary between objects of interest.

\section{Region Based Segmentation}

Region-based segmentation is used to look for similarities between adjacent pixels. That is, pixels that possess similar attributes are grouped into unique regions. The assumption is made that each region represents one object of interest. Using gray-level intensity is the most common means of assigning similarity, but many other possibilities exist, such as variance, color, and multispectral features.

\section{Clustering Based Segmentation}

The images are separated into various classes without any prior information. The data as similar as possible belong to same class and the data as different as possible belongs to different class.

\section{GENETIC ALGORITHM AND MORPHOLOGY FOR IMAGE SEGMENTATION}

The problem of detecting homogeneous regions in an image is addressed. We are concerned with identifying objects in a noisy image. As in a digitized image, the noisy image is represented by a matrix $\mathrm{X}$ with components, $\mathrm{Xij}$, whose value represents the intensity of the pixel $(i, j)$. The noisy image is produced by superimposing a noise component on the original image as described by the following equation:

$$
X i j=f i j+n i j
$$

Here, fij is the original image intensity and nij is the noise that is normally distributed with zero mean and variance ci. The original image in this study is composed of a single object intensity $\mathrm{R}_{\mathrm{o}}$ embedded in a background of intensity $R_{b}$. The segmentation algorithm is performed on $16 \times 16$ subimages, and the resulting subimages are then combined to obtain the entire segmented image.

The 2-D $16 \times 16$ subimage is represented as a vector m 1-D as

$\overrightarrow{\mathrm{x}}=[\mathrm{x} 0, \mathrm{x} 1, \ldots \ldots \ldots \ldots, \mathrm{x} 255], 0<\mathrm{xi}<255$ where $\mathrm{x}_{\mathrm{i}}$ represents the intensity of the pixel in the ith position.

$\begin{array}{cccccc}x_{0} & x_{1} & x_{2} & x_{3} & \ldots \ldots & x_{15} \\ x_{16} & x_{17} & x_{18} & x_{19} & \ldots \ldots & x_{31} \\ x_{32} & x_{33} & x_{34} & x_{35} & \ldots \ldots & x_{47} \\ \cdot & \cdot & \cdot & \cdot & \cdot & \cdot \\ \cdot & \cdot & \cdot & \cdot & \cdot & \cdot \\ \cdot & \cdot & \cdot & \cdot & \cdot & \cdot \\ x_{240} & x_{241} & x_{242} & x_{245} & \cdots \cdots & x_{255}\end{array}$

Fig. 1 The subimage matrix 


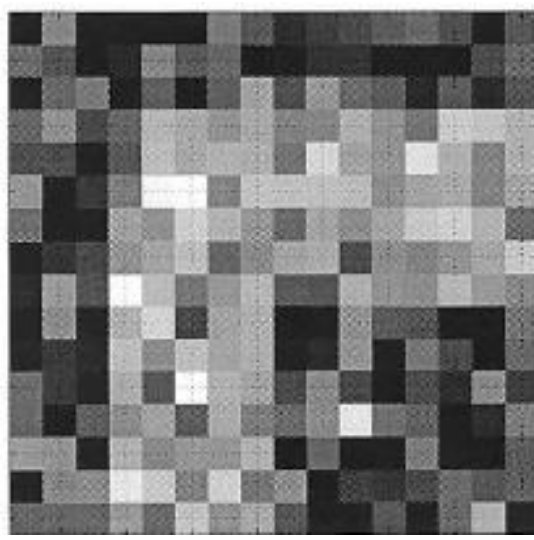

Fig. 2 2D 16x 16 noisy image

\section{Initial population}

This set is the initial population of GA. Each individual in this set is a string with element value of $R_{o}$ (objectintensity) or $R_{b}$ (background-intensity). The initial population can be represented by the set,

$$
\left\{Y_{\mathrm{i}}{ }^{\mathrm{k}}\right\}, \mathrm{i}=0,1, \ldots, 255 ; \mathrm{k}=1,2, \ldots, \mathrm{N}
$$

where $\mathrm{N}$ is the population size.

To generate candidates in the initial population, random method was used. That is, each element in the vector is randomly chosen to be $\mathrm{R}_{\mathrm{o}}$ or $\mathrm{R}_{\mathrm{b}}$.

$\left\{\mathrm{Y}_{\mathrm{i}}{ }^{\mathrm{k}}\right\}=\mathrm{R} 0$ or $\mathrm{Rb}$ (randomly), $\mathrm{i}=0,1, \ldots, 255 ; \mathrm{k}=1,2, \ldots$ , N

Each vector represents an individual in the initial population. These vectors actually are string representation of the subimage. Figure 3 shows a typical randomly generated 16x 16 subimage which is a candidate of the initial population.

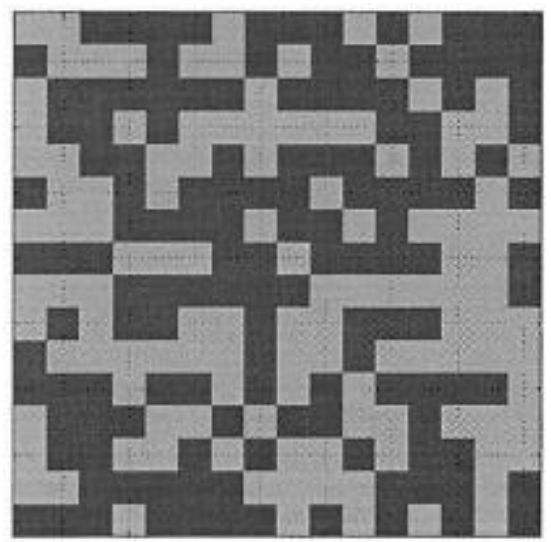

Fig. 3 candidate of initial population generated by random method

\section{Fitness evaluation}

The fitness function is very important in GA. It helps to decide whether the individuals are capable of producing offsprings, whether the offsprings are fit enough to survive, and whether the individuals in the current generation are capable of existing in the next generation.

If the original image is $\vec{x}$ and the initial population is

$$
\left\{\mathrm{Y}_{\mathrm{i}}{ }^{\mathrm{k}}\right\} \mathrm{i}=0,1, \ldots, 255 ; \mathrm{k}=1,2, \ldots, \mathrm{N}
$$

The fitness of an individual $\mathrm{f}(\mathrm{Yk})$ of the population is calculated as:

$$
\begin{gathered}
f\left(Y^{k}\right)=E\left(Y^{k}\right)+\alpha \times T\left(Y^{k}\right) \quad k=1,2, \ldots, N \\
\text { where } \quad E\left(Y^{k}\right)=\frac{1}{\sum_{i=0}^{255}\left|Y_{i}^{k}-x_{i}\right|} \\
T\left(Y^{k}\right)=\frac{1}{\sum_{i=0}^{255}\left|Y_{i}^{k}-w_{i}\right|} \quad w_{i} \in \text { neighboring pixel of } Y_{i}^{k}
\end{gathered}
$$

Two candidates along with the similarity measure value $\mathrm{E}\left(\mathrm{Y}^{\mathrm{k}}\right)$ and transition count measure $\mathrm{T}\left(\mathrm{Y}^{\mathrm{k}}\right)$ are shown. It can be seen that the candidate in Figure 4 is homogeneous and has a higher $\mathrm{T}\left(\mathrm{Y}^{\mathrm{k}}\right)$ than the one in Figure 5.

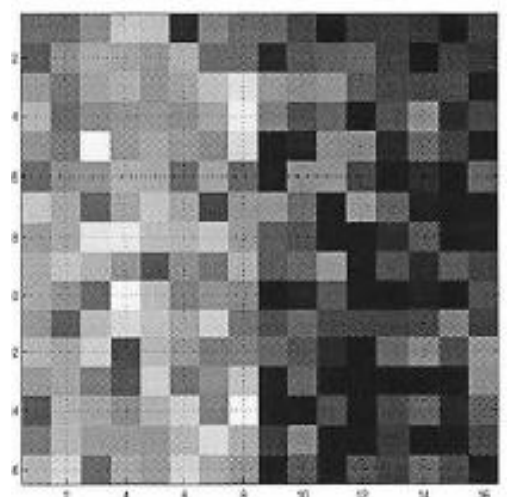

Fig. 4 16x 16 noisy image with SNR = 2

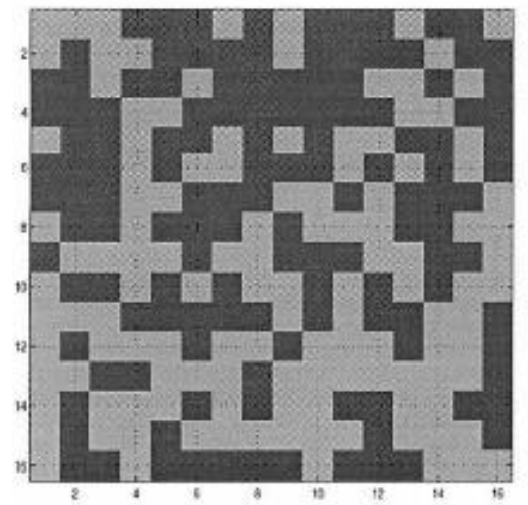

Fig. 5(a) Similarity: $E\left(Y^{k}\right)=5.6979 \times 10^{-5}$ Transition count: $\mathrm{T}\left(\mathrm{Y}^{\mathrm{k}}\right)=1.8904 \times 10^{-5}$

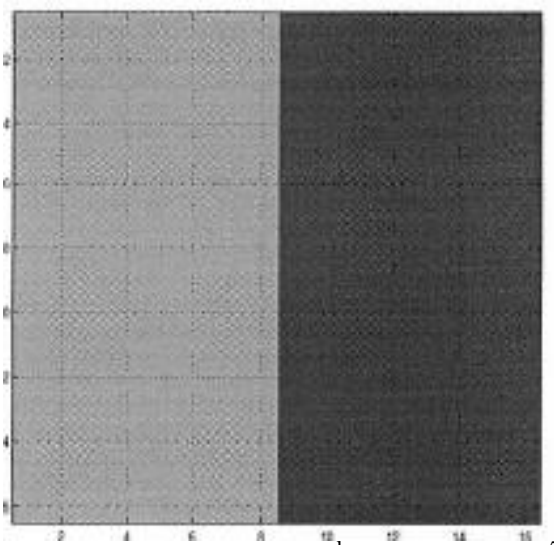

Fig. 5(b) Similarity: $\mathrm{E}\left(\mathrm{Y}^{\mathrm{k}}\right)=\stackrel{4}{9} .9140 \mathrm{u} \times 10^{\mathrm{t}}$ Transition count: $\mathrm{T}\left(\mathrm{Y}^{\mathrm{k}}\right)=1.0417 \times 10^{-4}$ 


\section{Selection of fittest individuals}

Once the evaluation of the fitness of the individuals has been done, the fittest individuals must be selected so that they can be mated to produce offspring in the next generation. A very simple way to accomplish the selection used in this thesis is by defining a threshold $\varphi$

$$
\Phi=\frac{\operatorname{Max}(f)+\operatorname{Min}(f)}{2}
$$

$\operatorname{Max}(f)$ and $\operatorname{Min}(f)$ are the maximum and minimum values of the fitness $f$, respectively. The individuals with fitness greater than the threshold are selected to produce offspring. Sometimes when ${ }_{\varphi}$ is small, in order that the search space does not get limited, a minimum number of offspring, $\eta$, is defined. If the number of generated offsprings is less than then $\eta$ the threshold ${ }_{\varphi}$ is decreased so that more individuals are selected for the reproduction and the number of offspring generated by the selected individuals is more than the minimum required.

\section{Reproduction}

This step is very important in the genetic algorithm because it is responsible for the evolution. The quality of final results depends on the GA operators in this step. The basic operators of reproduction, namely crossover and mutation. In this thesis, morphological operations are used as reproduction operators on selected individuals before the two-point crossover and mutation operators are applied.

\section{Morphological operations}

Morphological operation used in the first step is "closing" followed by opening. The structuring elements of size $3 \times 3$, $5 \times 5$, and $7 \times 7$ were tried. Depending on the particular problem, different structuring element size are chosen. The element value was also varied by assigning 50, 100, and 200 to the entire element. These two operations tend to clear small holes or small blocks in the noisy image and render the object and background regions more homogeneous. Individuals pre-conditioned using morphological operation are then subjected to crossover and mutation operators to generate offspring.

\section{Two-point crossover}

Based on Equation 5.5, only the individuals with fitness better than the threshold ${ }_{\varphi}$ considered fit to be parents. Two parents are randomly selected among these qualified individuals to perform a two-point crossover and produce two offspring.

Let $\mathrm{S}$ denote the set of $\mathrm{K}$ individuals selected from the current generation.

$$
S=\left\{Y_{j}^{i}\right\} \quad 1 \leq i \leq K, 0 \leq j \leq 255
$$

Every two individuals in the set $\mathrm{S}$ generate two new offspring as follows.

$$
\begin{gathered}
\left\{Y_{0}^{i}, Y_{1}^{i}, \ldots, Y_{p_{1}-1}^{i}, Y_{p_{1}}^{j}, Y_{p_{1}+1}^{j}, \ldots, Y_{p_{2}}^{j}, Y_{p_{2}+1}^{i}, Y_{p_{2}+2}^{i}, \ldots, Y_{255}^{i}\right\} \\
i, j=1,2, \ldots, K, \quad Y^{i}, Y^{j} \in\{S\}
\end{gathered}
$$

and

$$
\begin{gathered}
\left\{Y_{0}^{j}, Y_{1}^{j}, \ldots, Y_{p_{1}-1}^{j}, Y_{p_{1}}^{i}, Y_{p_{1}+1}^{i}, \ldots, Y_{p_{2}}^{i}, Y_{p_{2}+1}^{j}, Y_{p_{2}+2}^{j}, \ldots, Y_{255}^{j}\right\} \\
i, j=1,2, \ldots, K, Y^{i}, Y^{j} \in[S\}
\end{gathered}
$$

Among all this individuals, only fittest $\mathrm{N}$ (population number) of them is selected to survive.

\section{Mutation}

The reason that the mutation operator is introduced is because that, in nature, a living being undergoes some changes in its characteristics due to the influence of the surroundings. The mutation operations is intended to adapt the candidate better to its surroundings. The mutation operator is applied to those existing individuals in our study in a similar way in order to develop traits that make them fitter for survival. The mutation operator does not produce new offspring but it improves the fitness of the individual. For each bit $\mathrm{xi}$ in the individual $\mathrm{Y}_{\mathrm{i}}^{\mathrm{k}}$ the neighborhood $\mathrm{C}$ is defined and considered. It is similar to the 8-pixel neighborhood or 4-pixel neighborhood. If $\mathrm{N}_{\mathrm{b}}$ is the number of background pixels and No is the number of object pixels in $\mathrm{C}$ then

$$
Y_{i}^{k}=\left\{\begin{array}{ll}
R_{b} & \text { if } N_{b}>N_{o} \\
R_{a} & \text { if } N_{b}<N_{a}
\end{array} \quad i=0,1, \ldots, 255, \quad k=1,2, \ldots, N\right.
$$

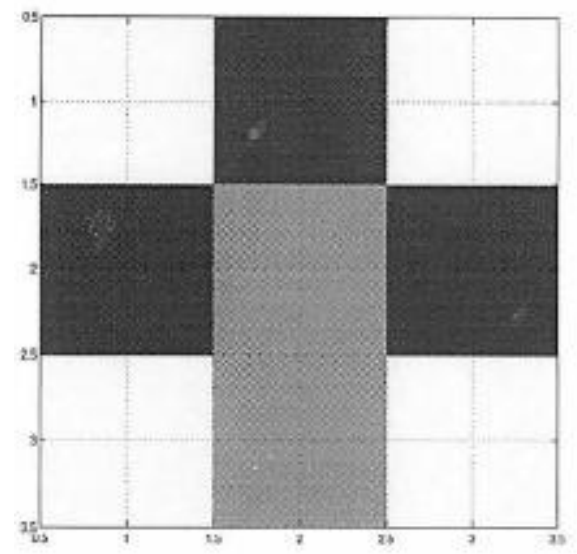

Fig. 6(a) 4-pixel neighbourhood

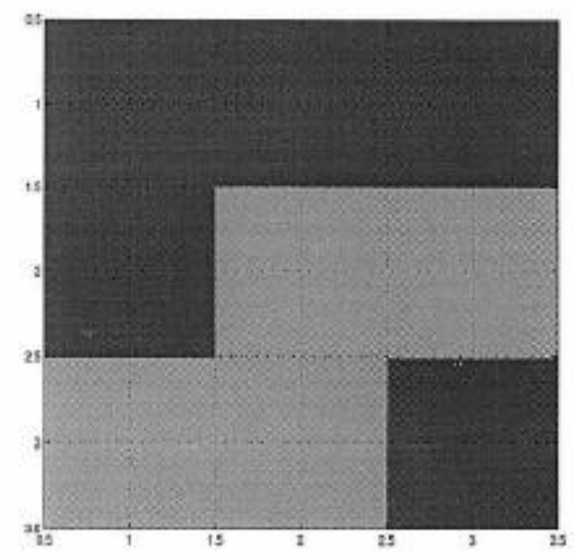

Fign. 6 (b) 8-pixel neighbourhood

\section{Stopping criterion}

The genetic algorithm evolves from one generation to the next generation and eventually, all individuals in the population will have the same (or very close) fitness after a 
certain number of generations, indicating that convergence is reached. Typically the algorithm will stop at this point because further processing will not improve the quality of the individuals. In this study, the algorithm is stopped either after a fixed number of generations or the maximum and minimum fitness of individuals are in a desired range.

\section{CONCLUSION}

An exhaustive parametric study of the algorithm on image segmentation was performed. The parameters that affect the performance of the algorithm were studied and discussed. The following observations can be made:

The initial population is generated randomly and no knowledge of the noise distribution is needed. The optimal population size in each generation is seen to be around 100 . The algorithm was terminated after 60 generations because the results do not show significant improvement after 50 generations. The optimal size of the structuring element is $5 \times 5$ for the $16 \times 16$ sub image with single object or two objects away from each other. But for small object or multiple objects which are close to each other, it is better to use a 3 × 3 structuring element. The new multi-point crossover technique gives better results than the two-point crossover technique. It also brings the diversity into the population. This is one of the reason that it gives better results. The algorithm is simple, fast and works very well on low signal-to-noise ratio image and on large size image.

\section{REFERENCES}

[1] Gonzalez, R.C. and Woods, R.E., Digital/mage Processing. AddisonWesley, Reading, Mass., 1992.

[2] Haralick, R.M. and Shapiro, L.G., "Image segmentation techniques," Computer Vision, Graphics, and Image Processing, vol. 29, pp.100-132, 1985.

[3] Pratt, W., Digital/mage Processing. 2nd edition, John Wiley \& Sons, New York, 1991.

[4] Horowitz, S.L. and Pavlidis, T, "Picture Segmentation by a Tree Transversal Algorithm," JACM, vol. 23, pp. 368-388, 1976.

[5] Rosenfeld, A. and Thurston, M., "Edge and Curve Detection for Visual Scene Analysis," IEEE Trans. Computers, vol. C-20, no. 5, pp. 562-569, May 1971.

[6] X.Z.Gao, -Soft Computing Methods for Control and Instrumentation,\| Institute of Intelligent Power Electronics Publications Espoo, pp.4, May 1999.

[7] M.S.Shyu, and J.J.Leou, , -A genetic algorithm approach to color image enhancement,Ill Volume 31, Issue 7, pp. 871-880, 31 July 1998.

[8] Dr. R.K Bhattacharjya, -Introduction To Genetic Algorithms, „IIT Guwahati, pp.12, 2012.

[9] Tao, C.V., 1998. Mobile Mapping Technology for Road Network Data Acquisition 1. Journal of Geospatial Engineering, Vol. 2, No.2, pp. 1-13. Tokyo, Japan.

[10] Robert, S.R.S., Haralick, M. and Zhuang, X., "Image analysis using mathematical morphology," IEEE Trans. Pattern Anal. Machine Intell., vol. 9, no 4, pp. 532-550, 1987.

[11] R.K. Mohanta, and B. Sethi, -A Review of Genetic Algorithm application for Image Segmentation,\| J.Computer Technology \& Applications, Vol 3 (2), pp.720-723, .

[12] B.Bhanu, and S. lee, and J. Ming, - Adaptive Image Segmentation using Genetic Algorithm, $\|$ IEEE Transactions on Systems, Man and Cybernatics, Vol 25(12), pp.1553, Dec 1995. 